

\title{
X-ray tomographic image post-processing and a new 2D LBM simulation for the determination of the porosity and the static airflow resistivity of an acoustic fibrous material
}

Pierre Lamary, Belisario N Huallpa, Flávio C Bannwart, Enio P de Deus, Ahmed Benallal, José R.F. Arruda

\section{To cite this version:}

Pierre Lamary, Belisario N Huallpa, Flávio C Bannwart, Enio P de Deus, Ahmed Benallal, et al.. X-ray tomographic image post-processing and a new 2D LBM simulation for the determination of the porosity and the static airflow resistivity of an acoustic fibrous material. Applied Acoustics, 2020, 169, pp.107452. 10.1016/j.apacoust.2020.107452 . hal-03103044

\author{
HAL Id: hal-03103044 \\ https://hal.science/hal-03103044
}

Submitted on 7 Jan 2021

HAL is a multi-disciplinary open access archive for the deposit and dissemination of scientific research documents, whether they are published or not. The documents may come from teaching and research institutions in France or abroad, or from public or private research centers.
L'archive ouverte pluridisciplinaire HAL, est destinée au dépôt et à la diffusion de documents scientifiques de niveau recherche, publiés ou non, émanant des établissements d'enseignement et de recherche français ou étrangers, des laboratoires publics ou privés. 


\title{
X-ray tomographic image post-processing and a new 2D LBM simulation for the determination of the porosity and the static airflow resistivity of an acoustic fibrous material
}

\author{
Pierre Lamary $^{\mathrm{a}}$, Belisario N. Huallpa ${ }^{\mathrm{b}}$, Flávio C. Bannwart ${ }^{\mathrm{c}, 1}$, Enio P. de Deus ${ }^{\mathrm{d}}$, Ahmed Benallal ${ }^{\mathrm{e}}$, José \\ R. F. Arruda ${ }^{f}$ \\ ${ }^{a}$ UFC, Department of Mechanical Engineering, Fortaleza, CE, Brazil \\ ${ }^{b}$ UFLA, Engineering Department, Lavras, MG, Brazil \\ ${ }^{c} U N I C A M P$, Department of Energy, Campinas, SP, Brazil \\ ${ }^{d} U F C$, Department of Materials Engineering and Metallurgy, Fortaleza, CE, Brazil \\ ${ }^{e} L T M$, ENS Paris-Saclay/CNRS/Université Paris-Saclay, France \\ ${ }^{f}$ UNICAMP, Department of Computational Mechanics, Campinas, SP, Brazil
}

\begin{abstract}
A set of X-ray tomographic images of a highly porous material composed of air-saturated coconut fibers is considered and used to estimate intrinsic characteristics of the material. Two physical properties are of interest: porosity and static airflow resistivity. For the porosity, two-dimensional gray-scale tomography images are obtained and post-processed to produce approximative black and white ones, unambiguously attributing distinct regions to fibers and air locations. The porosity is then directly deduced counting the black and white pixels. Several image processing algorithms are compared and associated porosities range from 0.76 to 0.97 , depending on the method employed, while it is estimated to be 0.86 from our analysis. For the airflow resistivity, the idea followed here is to use the pattern of the post-processed images as the lattice in a Lattice Boltzmann Method (LBM) fluid dynamics computation. To our knowledge, the LBM has not been used in this context before. A new 2D implementation of the method is therefore developed and studied. After tuning computational parameters, we have estimated the airflow resistivity using ten images of our sample to be $1382 \pm 12 \mathrm{~Pa} . \mathrm{s} / \mathrm{m}^{2}$. Both porosity and resistivity results are fully consistent with measurements obtained from a porosity-meter and a resistivity-meter, demonstrating the pertinence of X-ray tomography and the associated proposed methods.
\end{abstract}

Keywords: porous material characterization, JCA model, X-ray image thresholding, Darcy's law, Lattice Boltzmann Method, mesoscale simulation.

\section{Introduction}

Acoustic porous materials are generally defined as composed of solid and fluid phases. Waves propagating in the fluid are attenuated when passing 5 through the open pores due to viscous and thermal dissipation mechanisms, besides the structural dissipation due to the solid frame elastic deformation. This acoustic energy attenuation can be modeled using Biot's theory [1].

\footnotetext{
${ }^{1}$ Corresponding author at: Rua Mendeleyev, 200, Campinas, SP, CEP: 13083-860, Brazil. Email address:f cbannwart@fem. unicamp.br
}

10

A relevant aspect of Biot's theory is the homogenized equations. Allard 2 and other authors applied it to acoustic materials and proposed frequency domain dynamic equations coupling the fluid and structural phases. The reader can refer 15 to 3 for a recent review of acoustical methods for porous media or to [4 for a multi-physic approach to porosity. In the limiting cases where the solid frame is considered rigid or when its stiffness is considered negligible (limp models), an equiv20 alent fluid model can be alternatively used. For characterization purposes, these models are interesting since they include all acoustic dissipation mechanisms while still remaining simple. In this 
study, the equivalent fluid model applies without eters, the porosity and the static airflow resistivity, that can be characterized considering the static or quasi-static behavior of the material. The frequency tends to zero and, consequently, there is no vibration of the structural skeleton.

For the Biot-Allard's theory, the relevant aspect concerns the coefficients involved in the homogenized equations. They are expressed as frequency domain functions. Several models are available, and the Johnson-Champoux-Allard (JCA) micro-macro model is considered here. The JCA model involves five physical parameters: porosity, tortuosity, static airflow resistivity, viscous and thermal characteristic lengths. They are interrelated by two complex 40 valued equations (Eqs. 2 and 4), in which two parameters are known, especially the porosity and the static airflow resistivity. The remaining three parameters may be obtained experimentally, for instance using indirect methods, such as the ones in45 volving acoustic impedance tubes [5, 6, 7, or using inverse acoustical methods $8,9,10,11$. Other authors [12 [13] 14] 15] investigate theoretical, numerical and empirical models for the airflow resistivity involving a restrained set of parameters, such 50 as the fiber diameter and its influence on sound absorption coefficients, the fiber bulk modulus, and the porosity. In the works of [15], for instance, one important conclusion was that the nanofiber diameter of a nano-fibrous material selected for study,

55 varying from 80 to $397 \mathrm{~nm}$, was not a key factor in the determination of sound absorption coefficients. Nevertheless, such a conclusion does not discourage the investigations on airflow resistivities, as the conclusions may result completely different for this pa-

60 rameter. Our fibrous material, for instance, varies on its fiber diameter ranging from around $50 \mu \mathrm{m}$ to $300 \mu \mathrm{m}$, i.e, from the order of 1000 times larger than in the case of [15], which turns the problem much 110 different.

The main purpose of this work is to provide a new direct method of low computational cost to obtain the airflow resistivity of natural fibrous materials (intrinsically difficult to characterize and of sustain- 115 ability interest) from X-ray images. The driving 70 idea is to use the pixels of the image as the lattice of the Lattice Boltzmann Method. Besides, from the same X-ray images, we determine the corresponding porosity. Therefore, the work is essentially twofold. 120

The starting point is the utilization of a set of 75 X-ray Computer Tomography (CT) images of a fi- brous acoustic material to estimate its porosity (direct method). The fibers are natural and were obtained from the outer skin of coconuts. They were sprayed with latex and compressed to form a fibrous material [16. To process the resulting pile of gray scale CT images, we apply the method proposed in [17, where an algorithm attributes only black or white color to each image pixel and generates a data histogram. Next, the post-processed image is utilized to carry out $2 \mathrm{D}$ simulations with the airflow resistivity as indicator, also consisting in a direct method.

From a general point of view, experimental test rigs consist of two main parts: physical devices o equipped with sensors, and numerical procedures to transform the sensor information into meaningful indicators. In this study, the physical device is an $\mathrm{X}$-ray tomography equipment, and the numerical procedures are subsequent image post-processing 5 and 2D LBM simulations to estimate, respectively, the porosity and the static airflow resistivity of a porous sample. The originality and the novelty of the work come from the fact that, to our knowledge, the numerical procedures suggested here have not been presented in the literature in the context of acoustic characterization. In particular, for what concerns the LBM method, the closest related works were found in the field of soil mechanics with the determination of the permeability of low porous rocks saturated with liquid. Another related field where advanced mesoscale calculation are carried out is the field of homogenization; and we may cite the works of [18, 19].

This paper is organized as follows: We first describe the investigated porous material and the experimental procedures; then, we present the acoustic indicators relative to the estimated ones following the Biot-Allard theory of poroelasticity; we then propose the numerical methods to estimate the porosity based on statistical techniques and the static airflow resistivity using the LBM method applied to fluid dynamics; the results obtained with these proposed methods are then compared with experimental values obtained directly using a porosity-meter and a resistivity-meter. Discussions of the results are provided all along the paper and in the conclusion. 


\section{Materials and experimental procedures}

\subsection{Materials}

The specimen used in this work was cut from a board made of coconut fiber recovered with natural latex produced by a local manufacturer (Coquim, São Paulo, Brazil). The sample size $\left(50 \times 50 \times 50 \mathrm{~mm}^{3}\right)$ was selected in order to preserve the characteristics of the original arrangement of the compacted fibers and to allow measurement in acoustic devices. The sample is shown in Figure 1 .



Figure 1: Porous sample under study.

\subsection{X-ray tomography}

The X-ray tomography equipment used in this study is the model X50 (Fig. 2) from the North Star Imaging Company, Inc.. It consists of a protected radiation cabin, 5-axis manipulator, dual $\mathrm{X}$-ray tube of $225 k \mathrm{~V}$ with dual source (reflection and transmission), and a detector DEXELA 2923, CMOS image sensor technology with scintillator and interface options, plane cesium 3072 x 3888 pixel matrix, 14-bit and 26 frames/second at full resolution. A software suite manages the data acquisition, image processing and $3 \mathrm{D}$ reconstruction. In this work, the molybdenum tube was set at $50 \mathrm{kV}, 150 \mu \mathrm{A}$ and $23.1 \mathrm{~W}$. The $14002 \mathrm{D}$ slices perpendicular to axis $y$ (across the thickness of the sample) were acquired at the average velocity of 10 frames/second.

Fig. 3 shows a 3D image reconstruction of the sample of Fig. 1 provided by the software suite of the X-ray tomography equipment.

\subsection{Porosity-meter and resistivity-meter}

In order to get reference values, our sample was



Figure 2: X-ray tomography equipment.



Figure 3: Tomographic 3D image reconstruction of the porous sample under study.

i) PHI - Porosity and Density Meter and the ii) HF SIGMA - High Airflow Resistance Meter developed by Mecanum Inc..

\section{Porous material parameters}

In this section, the JCA and fluid equivalent models are introduced to recall and show how the porosity and the resistivity parameters are embedded in the formulation. It will be therefore needed to solve the fluid equivalent model equation in order estimate the sensitivity of the material parameters toward the global acoustic indicators such as the absorption and the transmission losses through the porous sample. This will be done in the discussion of paragraph 5.6 
The fluid equivalent model is generally obtained by a limiting process from the poro-elastic equations, in particular, from the so-called $(U, p)$ formulation. The reader will find many documents, as cited in this section, following this approach. To renew with the writing of this model and to give another physical insight, the fluid equivalent model is introduced here from the Helmholtz equation of acoustics.

\subsection{Biot theory}

Biot introduced homogenized equations for the modeling of the behavior of poroelastic and porous materials [1. These equations can be written in the frequency domain and coupled with structural dynamics and acoustics. Implemented in software based on semi-analytical methods such as the Transfer Matrix Method [20, 2, 21, 22, 23, 7], or based on numerical methods such as the Boundary Element Method 24 and the Finite Element Method [2, 25, 26], the Biot equations allow for the study of complex acoustic insulation systems. Without loss of generality, let us consider the equivalent fluid model, which gathers all the dissipative phenomena of interest here. The fluid consists of the fluid that can go through the open pores; the skeleton is motionless. Rigid motion of the skeleton can be taken into account if needed for coupled problems, where the fluid-structure dynamic interaction is relevant; this model is referred to as equivalent fluid limp model.

From substitution of the behavior laws (stressstrain relations) in the dynamic motion equations, and using the acoustic pressure $\tilde{p}$ as the main unknown, the fluid equivalent model reads as the following Helmholtz equation:

$$
\Delta \tilde{p}+\tilde{k}^{2} \tilde{p}=0,
$$

where $\tilde{k}$ is a lossy wavenumber, designated complex by the tilde notation $(\sim)$, employed throughout the text.

For an acoustic ideal fluid (without viscous losses or thermal relaxation) the differential equation (1) applies, and the classical acoustic equation is recovered, with $\tilde{k}$ a real wavenumber defined as $\tilde{k}=k=$ $\omega / c$, where $\omega$ is the angular frequency and $c$ the speed of sound. Associated boundary conditions are, $\tilde{p}=\bar{p}$, an imposed pressure at a given boundary, and $\partial \tilde{p} / \partial n=\rho_{0} \omega^{2} \bar{u}_{n}$, an imposed normal acoustic displacement at other boundaries, where $\rho_{0}$ is the fluid density. If $\bar{p}$ and $\bar{u}_{n}$ are real, $\tilde{p}$ becomes therefore real as well. Internal dissipation can be introduced in this equation considering, for instance, a damping factor $\eta$ applied to the acoustic bulk modulus $K_{0}$ as $\tilde{K}=K_{0}(1+j \eta)$ (viscoelastic model), changing the definition of $c=\sqrt{K_{0} / c}$ to a complex speed of sound $\tilde{c}=\sqrt{\tilde{K} / \rho_{0}}$ and leading finally to a complex wavenumber $\tilde{k}=\omega / \tilde{c}$.

The Biot equivalent fluid model generalizes these definitions with $\tilde{c}(\omega)=\sqrt{\tilde{K}(\omega) / \tilde{\rho}(\omega)}$. When this model is considered to occupy the entire porous material, i.e. all the volume of the fluid in the pores and of the skeleton, the equivalent pertinent entities are $\tilde{K}_{e q}(\omega)=\tilde{K}(\omega) / \phi$ and $\tilde{\rho}_{e q}(\omega)=\tilde{\rho}(\omega) / \phi$, where $\phi$ is the porosity. For instance, $\partial \tilde{p} / \partial n$ at the boundary has to be written $\partial \tilde{p} / \partial n=\tilde{\rho}_{e q}(\omega) \omega^{2} \bar{u}_{n}$.

\subsection{The JCA micro-macro model}

The micro-macro models provide formulae to express $\tilde{K}(\omega)$ and $\tilde{\rho}(\omega)$. Several micro-macro models are presented in 2. The simplest one is the Delany and Bazley model, which only requires the knowledge of the static airflow resistivity. Others are dedicated to specific pore geometries. One of special interest is the Johnson-Champoux-Allard (JCA) model, which gives good predictions for random pore distribution and is commonly adopted today.

In this model, $\tilde{\rho}(\omega)$ and $\tilde{K}(\omega)$ can be defined according to the formalism of 27 as:

$$
\tilde{\rho}(\omega)=\alpha_{\infty} \rho_{0}\left(1+\frac{1}{j \hat{\omega}} \sqrt{1+j \tilde{G}(\omega)}\right),
$$

with

$$
\tilde{G}(\omega)=\sqrt{1+j \frac{M}{2} \tilde{\omega}}
$$

where $\tilde{\omega}=\frac{\omega \alpha_{\infty} \rho_{0}}{\phi \sigma}$ is a dimensionless frequency, and $M=\frac{8 \alpha_{\infty} \rho_{0}}{\phi \Lambda^{2} \sigma}$ is a shape factor. And

$$
\tilde{K}(\omega)=\frac{K_{0}}{\gamma-(\gamma-1)\left(1+\frac{8 \eta}{j \omega \rho_{0} N_{p r}^{2} \Lambda^{\prime 2}} \tilde{G}^{\prime}(\omega)\right)^{-1}}
$$

with

$$
\tilde{G}^{\prime}(\omega)=\sqrt{1+j \frac{\omega \rho_{0} N_{p r}^{2} \Lambda^{\prime 2}}{16 \eta}}
$$


The known entities are $\rho_{0}, K_{0}, N_{p r}$ and $\gamma$ - air thermophysical properties. $N_{p r}$ is the Prandtl number set to 0.71 , and $\gamma$ is the ratio of specific heats taken to be 1.4. The JCA parameters are labeled $\phi, \sigma, \alpha_{\infty}, \Lambda$ and $\Lambda^{\prime}$, respectively: porosity, static airflow resistivity, and viscous and thermal characteristic lengths. From Eq. 2 one can note that $\tilde{\rho}(\omega)$ involves only the first four parameters of the model 305 and is linked to viscous effects, while, from Eq. 4 $\tilde{K}(\omega)$ involves the fifth and last parameter, linked to thermal effects.

\section{Estimating porosity from XRCT images}

\subsection{Porosity}

Porosity is defined as the ratio of the volume occupied by the fluid phase (usually air) to the total porous material volume, which also embraces the solid frame phase [2]. Porosity must be measured or computed for a representative volume of the acoustic material. A representative volume has a characteristic length much smaller than the wavelength of the waves in the audio frequency range and much larger than the random microscopic features of the material, so that average properties of the homogenized material can be obtained.

Traditional methods usually applied to experimentally estimate porosity can be classified in two types. Direct methods consist in the immersion of a porous material sample in a incompressible fluid (typically water) and computing the volume ratio between the fluid phase and the entire sample 28 . Otherwise, if the mass density of the solid phase is known and homogeneous, from the weight of the sample, the volume of the solid frame can be computed and subtracted from the total volume obtained from the sample external geometry. More precise methods are based upon gas expansion in cavities with known volume and pressure measure- ${ }^{335}$ ments, such as in the Boyle's Law Porosimeter 29.

\subsection{X-ray $C T$ 2D images}

The directly obtained X-ray CT images reveal $2 \mathrm{D}$ transversal slices of a sample 30 , sequentially numbered. Each image is formed according to the $\mathrm{X}$-ray attenuation dependency on the local material density through which the radiation is transmitted. Using appropriate image processing transformations, it is possible to create a 3D image from slices of a material sample. For high energy X-rays (above $100 \mathrm{kV}$ ) [17, those images are associated to the so-called CT number and correlated to mass density. Therefore, in principle it is possible to estimate the porosity of a sample of porous material using CT images. If an acoustic material is composed of solid and fluid (air) phases, the solid phase is likely to have the higher density, which makes it distinguishable by contrast. Evaluation of the proportion between phases leads to determine the volume of air as a percentage of the total volume, i.e. the porosity. Usual CT images are calibrated so that water gives zero for its CT value, and air gives -1000 . The solid phase has values typically larger 310 an zero.

In acoustics, we are interested in the open porosity, i.e., the domain of the material where an external fluid can flow through. Therefore, closed pores, where a fluid has been imprisoned when making the material, have to be excluded. 2D images may artificially produce closed pores. However, from the nature of our material, made of fibers, those pores are likely to be open when the 3D situation is considered. Thus, we can count all the fluid part in the $2 \mathrm{D}$ images as open pores (white).

\subsection{Thresholding}

The major problem for estimating porosity from CT images is the segmentation process (thresholding) in order to separate air from solid. Visual thresholding is prone to subjective errors and lacks repeatability. A recent work by 17 proposes a method based upon CT images to estimate porosity. Given a set of images constituting the scan of a representative volume of the porous material, to each pixel (or voxel, in the 3D case) of the image, a CT number ranging from 0 to $r_{\max }$ is attributed. They are typically gray scale image integer values. The range of $\mathrm{CT}$ values is divided into bins, and a histogram is computed, so that to each range of values $r_{i},(i-1) \Delta r<r_{i}<i \Delta r$, has a count of $n_{i}$ in a total number of pixels (or voxels) of $n$, with $H\left(r_{i}\right)=n_{i} / n$ and $\sum_{r_{i}=0}^{r_{i}=r_{\max }} H\left(r_{i}\right)=1$. In this case the porosity can be estimated with:

$$
\Phi\left(r_{\max }\right)=\frac{\sum_{r_{i}=0}^{r_{i}=r_{\max }}\left(r_{\max }-r_{i}\right) H\left(r_{i}\right)}{\sum_{r_{i}=0}^{r_{i}=r_{\max }} r_{\max } H\left(r_{i}\right)} .
$$

This value is computed for different values of $r_{\max }$, and the minimum of the obtained curve corresponds to the desired porosity. Depending on how the image is processed, the value of $1-\Phi$ can be obtained, as it is the case in the example treated in 
this work. The results obtained with this method 380

the later on as "Our analysis") are compared with the classical methods based on the thresholding approach, such as IsoData 31, Huang 32, Li 33, Mean 34, Moments 35, Otsu 36, and Shanbhag [37, as shown in Table 1. These thresh- 385 olding methods are available in the public open domain ImageJ program (www.imagej.net) used in this work.

\subsection{Porosity estimation results}

A coconut fiber sample measuring approximately $50 \times 50 \times 50 \mathrm{~mm}^{3}$ was placed in a X-ray CT, and 1158 slices were measured. We have observed that the slices with low data acquisition noise, slightly apart from the cut surfaces, were numbered between 150 and 939, which means 790 slices. After selecting the slices and applying a crop process, a 3D cube with dimensions of $40 \times 40 \times 40 \mathrm{~mm}^{3}$ and with voxel size of $46.1 \times 46.1 \times 46.1 \mu \mathrm{m}^{3}$ was selected.

Figure 4 shows the X-ray CT cropped 3D image of the coconut fiber material. The initial specimen size of $50 \times 50 \times 45 \mathrm{~mm}^{3}$ (Fig. 3) was reduced to a final size of $40 \times 40 \times 40 \mathrm{~mm}^{3}$.

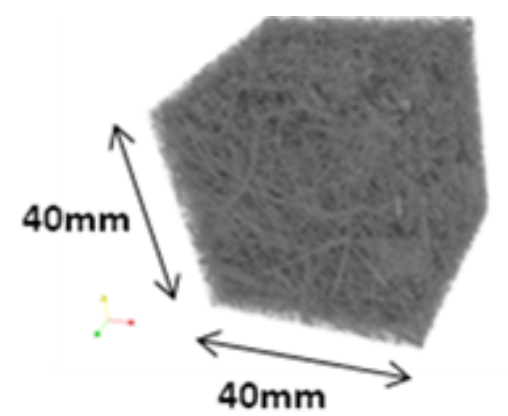

Figure 4: XRCT 3D cropped image.

The dimension of a slice of this 3D image is therefore $40 \times 40 \mathrm{~mm}^{2}$ with a pixel size of $46.1 \times 46.1 \mu \mathrm{m}^{2}$. 390 The thickness is $46.1 \mu \mathrm{m}$. In the following, we will indifferently call a slice of unit pixel thickness, a slice or a 2D image. Figure 5 shows a slice image. The clear spots correspond to the denser material (fiber), while the dark gray are the pores (fluid). 395 Then, the 2D images were submitted to the thresholding process (transformation of the gray scale image to binary scale) using different methods that software imageJ offers. The methods used were IsoData, IJ-IsoData, Li, MaxEntropy, Mean, Mo- 400 ments, Huang, Otsu, and Shanbhag. After selecting the threshold method (binarization), which permits the conversion to black and white pixels, the porosity can be computed. Black pixels are associated with the skeleton and white pixels to the fluid. The porosity is given by the ratio of the number of white pixels and total of number of white and black pixels. Figure 6 shows the result obtained when using the Otsu's thresholding method.



Figure 5: Original image.

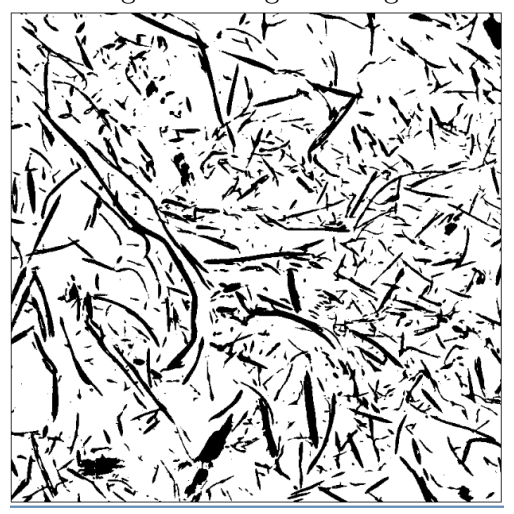

Figure 6: Processed image.

Table 1 shows the results of the porosity values for the different threshold methods. It can be observed that different threshold methods yield different values for porosity. The values of the porosity present a range of variation between 0.76 and 0.96 , which means a dispersion of 0.2 . Huang, Li, and Mean methods present lower values, in opposition to Moments and Shanbhag methods. The value of 0.87 , labeled as RefTest (Reference Test), is the one obtained when using a porosity-meter.

To apply the histogram-based grey scale image method proposed by [17, the CT image was read within MATLABC and a histogram with 100 bins was built, as shown in Fig. 7. The resulting porosity 
Table 1: Results of porosity using different thresholding methods.

\begin{tabular}{|c|c|c|c|}
\hline Method & Our analysis & Huang & Isodata \\
\hline Porosity & 0.86 & 0.78 & 0.83 \\
\hline \hline Method & Max Entropy & Mean & Moments \\
\hline Porosity & 0.82 & 0.78 & 0.93 \\
\hline \hline Method & Li & Otsu & Shanbhag \\
\hline Porosity & 0.78 & 0.85 & 0.97 \\
\hline \hline Method & RefTest & & \\
\hline Porosity & 0.87 & & \\
\hline
\end{tabular}

values obtained for the first slice (number 150) are shown in Fig. 8. Being restricted to one slice, this plot is not conclusive, as many local minima are present. Proceeding with the computation of this curve for all 790 slices of the sample and averaging, as presented on the plot of Fig. 9, the porosity can be estimated to be 0.86 . When compared to the others methods (Table 1), our analysis provides the closest value to the measured one of 0.87 (RefTest).

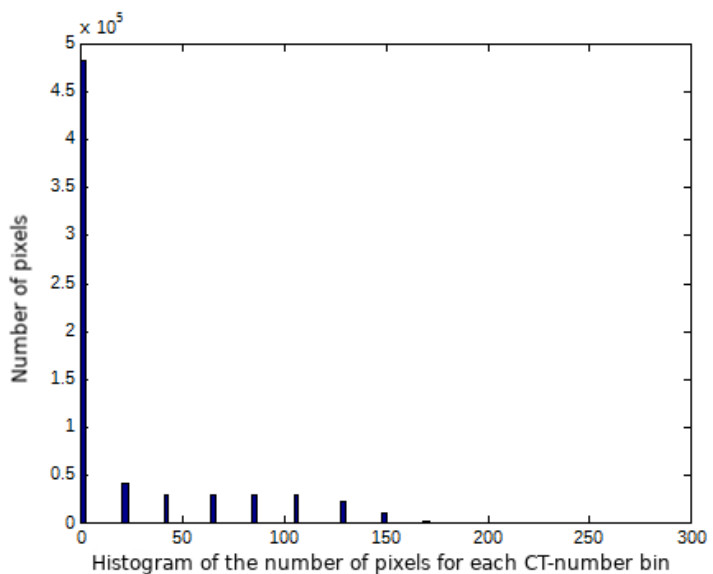

Figure 7: Tomographic 3D image reconstruction of a porous sample under study.

\subsection{Discussion}

The proposed method draws benefit from the detailed information coming from the tomographic images , i.e., it uses all pixels constituting the volume of the sample. Doing this, and as shown by the results obtained, the method allows the accurate prediction of the porosity of the sample.

Furthermore, the different thresholding methods used to estimate porosity can produce the black and



Figure 8: Estimating porosity from one slice with the method proposed in 17 .

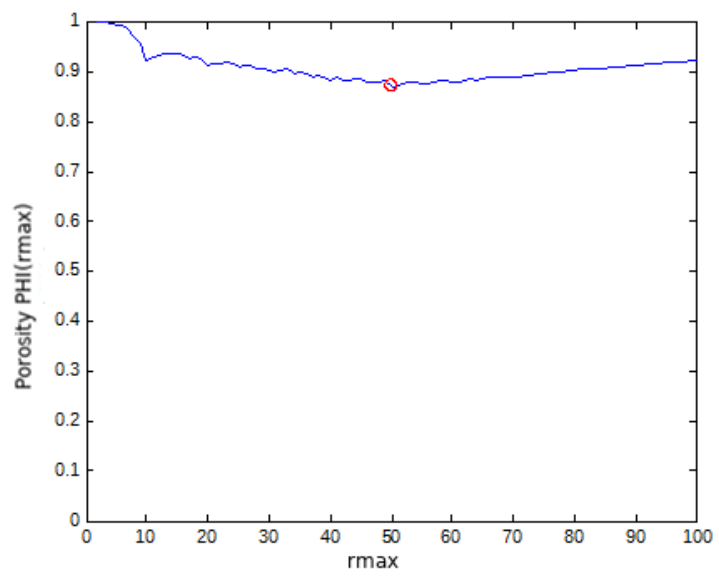

Figure 9: Estimating porosity from 790 slices with the method proposed in [17. following section concerning the use of the LBM method to estimate the resistivity of the sample. Therefore, it is suggested to use the best suitable alternative thresholding method, which yields the closest value to the proposed method, to produce black and white images of the sample. From the analysis results synthesized in Table 1, the best suitable candidate is the Otsu's method, which will be used in the following section. 


\section{Estimating the airflow resistivity using 2D LBM simulation}

Semi-analytical phenomenological micro-macro models, such as the JCA model, are developed considering the Poiseuille type flow, which is the type of nar flow). However, the objective of this work is not to completely substitute the micro-macro model by LBM calculations - since it would require the consideration of the coupling factor and parameters volved in the frame of Biot theory as frequency functions, an entanglement beyond our interest for now. Instead, the objective here is determination of just one of the five parameters needed to fulfill the JCA model, which is the static airflow resistivity.

${ }_{445}$ This parameter is obtained for very slow airflow. In this regime, the velocity of the airflow is proportional to pressure drop along the sample leading to a constant value for the resistivity, i.e., independent of the velocity of the flow. Classical experimental methods to determine the airflow resistivity use a resistivity-meter $[22]$.

\subsection{Darcy permeability and airflow resistivity}

When fluid goes slowly through pores, resistive phenomena induced by viscous friction are predominant and those forces balance with the fluid velocity. The pertinent parameter to describe this phenomena is the permeability of the medium, which is expressed by the Darcy's law. Written in an upto-date form, the permeability $\boldsymbol{k}$ is a second order tensor that we may write in Cartesian coordinates as

$$
k_{i j}=-\frac{\eta}{p_{, j}}\left\langle v_{i}\right\rangle
$$

where $v_{i}$ is the outflow speed field in the $i$ direction, \langle\rangle is the volume average, $\left\langle v_{i}\right\rangle=\phi \frac{\int_{A} v_{i} d a}{\int_{A} d a}$, with $\phi$ being the porosity and $A$ being the area in $2 \mathrm{D}$ (or he volume in $3 \mathrm{D}$ ) of the fluid part, and $p_{, j}$ is the gradient pressure in the $j$ direction. This gradient applies as a field all over the wet fluid domain.

From this, the static airflow resistivity $\sigma$, which is also a second order tensor, can be related to $\boldsymbol{k}$ as

$$
\sigma_{i j}=\eta\left(k_{i j}\right)^{-1}
$$

An acoustic material is generally manufactured in layer form, and the fabrication process may turn 505 the layer orthotropic. If its principal directions are priorly known, tests or computation shall be carried out in those directions to directly express Eqs. 8 and 75 9, for instance. The permeability and resistivity tensors also possess the propriety of symmetry. The reader can refer to 38, where experimental and numerical procedures are presented to characterize anisotropic materials.

Neglecting the non-diagonal terms of $\boldsymbol{k}, \mathrm{Eq} \cdot 8$ can be solved as

$$
\sigma_{i i}=\frac{\eta}{k_{i i}}
$$

with $i \in\{1,2\}$ for our $2 \mathrm{D}$ simulations. The mean value of the tensor defined as $\sigma=\left(\sigma_{11}+\sigma_{22}\right) / 2$ is considered as our final indicator. To recover the $3 \mathrm{D}$ situation, several slices of a sample are considered, each slice being a $2 \mathrm{D}$ image treated by a $2 \mathrm{D}$ calculation. Statistic mean values are given to approximate the global resistivity of the $3 \mathrm{D}$ sample.

\subsection{The numerical model}

The numerical model is inspired by the resistivity-meter configuration and is sometimes called numerical tube. It consists, see Fig. 10, in the $2 \mathrm{D}$ image sample to be computed, two side impervious rigid walls, and two fluid buffers: inlet and outlet. The dimensions are given in number of pixels. For the analysis presented further on, the $2 \mathrm{D}$ images are of $821 \times 821$ pixels (each pixel sized $46.1 \times 46.1 \mu \mathrm{m}^{2}$ ), and the remaining parts are two pixels thick. A pressure gradient, oriented in the longitudinal direction of the tube (direction 1 , in Fig. 10 is imposed along the entire domain of the model, and the LBM algorithm computes iteratively the corresponding velocity field, from which, the resistivity (in this direction) is deduced. To get the resistivity in the direction 2 , the porous sample is simply rotated at an angle of $90^{\circ}$.



Figure 10: 2D numerical model.

From a technical point of view, the LBM algorithm implemented in this work refers to the LBM-BGK method with D2Q9 cells and a direct 
bounce-back scheme. Bounce-back occurs when the fluid in question meets a rigid obstacle such as the e walls and the fibers of the material. These choices correspond to one of a most simple implementation of LBM. The reader can refer to Appendix A to access the algorithm we used in this study. The full script of the program is also available and can be found in the following repository (http:www.labvib.ufc.br/CavLBM.html).

\subsection{Physical units and LBM units}

\subsubsection{Basic conversions}

Many studies using LBM concern the enhance-

ment of the method, and their results are presented in Lattice units. To solve physical problems, physical units shall be converted to Lattice units and, next, shall express the LBM results in physical units. The conference paper 39 focuses on this aspect and presents a clear methodology. Conversion factors are established for the fundamental entities, L (length), M (mass), and $\mathrm{T}$ (time). Conversion for secondary entities such as pressure or velocity are deduced from the previous factors.

530 Three independent primary conversion factors are needed, chosen to be, in our case, length, dynamic viscosity and density. Figure 11 is extracted from our MATLABC code; the 2D image has a resolution of $\left(\mathrm{NX}^{*} \mathrm{NY}\right)$ pixels and the pixel size is

535 known. "Phys" refers to physical units, and "Lat" to Lattice units. The pre-process to LBM algorithm (Physical to LBM units) and the post-process to LBM algorithm (LBM to Physical units) are shown. The LBM algorithm itself is omitted.

\section{5.3.2. Advanced conversion: $2 D$ to $3 D$ resistivity}

As shown in Fig. 11, the last instruction concerns deriving a $3 \mathrm{D}$ resistivity from the $2 \mathrm{D}$ computation. Indeed, the 2D situation does not correspond to the real 3D situation of Fig. 4. It corresponds to a 3D situation where the $2 \mathrm{D}$ pattern repeats itself in the third direction; velocities are compelled to stay inplane.

Therefore, an ad hoc corrective formula is used where it is considered that a fluid particle can escape in the direction 3 constrained by a pattern identical to the one of the direction 2. For a square cylinder, the following relation can be written $\left\langle\left|v_{t}\right|\right\rangle=\sqrt{\left\langle\left|v_{2}\right|\right\rangle^{2}+\left\langle\left|v_{3}\right|\right\rangle^{2}}$, with $v_{t}$ being the velocity of the particle in the $(2,3)$ planes, and $v_{2}$ and
\% From physical to LBM units

Phys_H $=$ NY*PixelSize \% $(\mathrm{m})$

Phys Dyn Visco $=1.84 \mathrm{e}-5 \%(\mathrm{~Pa} / \mathrm{s})$

Phys_Density $=1.225 \%(\mathrm{~kg} / \mathrm{m} 3)$

Phys Gravity $=9.81 \%(\mathrm{~m} / \mathrm{s} 2)$

Phys_Kin_Visco $=$ Phys_Dyn_Visco/Phys_Density $\%(\mathrm{~m} 2 / \mathrm{s})$

Lat_H $=\mathrm{NY}$

Lat Density $=1.0$

Lat_Tau $=0.66 \%$ chosen by the user $\%$

\%Conversion: Phys_Q = Lat_Q x Coef_Q

Coef_H $=$ Phys_H/Lät_H

Coef Density = Phys Density/(Lat Density)

Lat_Kin_Visco $=($ Lat_Tau -0.5$) / 3.0$

Coef $\mathrm{t}=$ Lat_Kin_Visco* Coef_H ${ }^{*}$ Coef_H / Phys_Kin_Visco

$\%$ Coef deduced

Coef_u $=$ Coef_H/Coef_t

Coef $\mathrm{F}=$ Coef Density*Coef $\mathrm{H}^{\wedge} 4 /$ Coef $\mathrm{t}^{\wedge} 2$

$\%$

Phys $\mathrm{dPdL}=20.0 \%(\mathrm{~Pa} / \mathrm{m})$ chosen by the use Lat_dPdL $=$ Phys_dPdL/Coef_Density/Coef_H*Coef_t*Coef_t

LBM algorithm

\%From LBM to physical

Phys2_K0 = Coef_H*Coef_H*Lat2_K0_Darcy_Porous \%(m2)

Phys2_Res = Phys_Dyn_Visco / Phys2_K0 \%(Pa.s/m2)



Figure 11: Pre and post process for units conversion.

$\left\langle\left|v_{t}\right|\right\rangle=\sqrt{2}\left\langle\left|v_{2}\right|\right\rangle$, with $\left\langle\left|v_{2}\right|\right\rangle=\phi \frac{\int_{A}\left|v_{2}\right| d a}{\int_{A} d a}$ being the mean of the module of the velocity in that direction. As the permeability can be sighted as an effective area, which resumes to a line for 2D calculations, it is suggested to modify this effective length line from a factor of $\sqrt{2}$. Therefore, in the following study, the $3 \mathrm{D}$ resistivity is deduced from the $2 \mathrm{D}$ calculations with the following formula: $\sigma_{3 D}=\frac{\sigma_{2 D}}{\sqrt{2}}$.

Establishing a general empirical or mathematical formula would be an interesting topic of investigation, however out of the scope of the present study. We would like to point out that any general formula could be simply analyzed numerically, comparing $3 \mathrm{D}$ and $2 \mathrm{D}$ calculations and generating random samples in order to cover the range of interest of the technical parameters of the acoustic materials. Concerning the porosity, which is recalled and indicated in the coming slice by slice analysis (see

575 Table 3 and Table 4), it can be noticed that once the volume is discretized into voxels, the porosity can be evaluated slice by slice. In order words, at the difference of the resistivity, the porosity is independent on the size of the problem. 


\subsection{Numerical convergence}

The size of the model (the lattice) is imposed, in our case, by the resolution of the image, given in pixels, and by the pixels dimension. From this, the remaining parameters to be set by the user are the relaxation time and the value of the pressure gradient. Calculation is followed looking at the evolution of the software tolerance $\mid V_{1}($ iter -1$)-$ $V_{1}($ iter $) /\left(V_{1}(\right.$ iter -1$) \mid$, where $V_{1}$ is the mean value of the macroscopic velocity of the fluid phase in the direction 1, and following up the convergence of our main indicator, the 3D resistivity. Images of the airflow are from time to time examined.

\subsubsection{Relaxation parameter}

Convergence is typically of three types: i) an ${ }^{62}$ overshoot of the converged value and next decreasing oscillations towards the converged value, ii) a convergence from upper or lower values with or without weak oscillations, iii) spurious behavior with a phase with no convergence followed neither convergence nor divergence. The latter behavior was not observed. When divergence occurred, it happened within the first 1,000 iterations over the 20,000 iterations generally considered. Figure 12 presents typical convergence curves obtained when



Figure 12: Convergence curves for different relaxation time values.

\subsubsection{Pressure gradient}

Pressure gradient is input in physical units. When water gravity problems are considered (as Darcy did with a column of water going through

sand), the pressure gradient is therefore $\vec{\nabla} p=$ $\rho \vec{g}$ and leads to an intensity of $1000 \times 9.81 \approx$ $10000\left(\mathrm{~N} / \mathrm{m}^{3}\right)$. If an air column is considered the intensity of the body force is $1.225 \times 9.81 \approx$ $10\left(\mathrm{~N} / \mathrm{m}^{3}\right)$. The calculations are explored in this range and next extended to lower values. The very question is to stay in the domain of validity of LBM-BKG, which also fulfills the resistivity definition. In order words, the static airflow resistivity has to remain constant for quasi-static calculations. Fig. 13 shows the results obtained on a given sample, all calculations are conducted with $\tau=0.66$ and up to 20,000 iterations, except for the last point, $\vec{\nabla} p=1000$, where it was necessary to tune $\tau$ to 0.55 to have convergence. For further analyses, the couple of inputs $(\tau, \vec{\nabla} p)$ are chosen to be $(0.66,20)$ to satisfy the hypothesis and to produce convergence within 20,000 iterations.



Figure 13: Adjusting of $\vec{\nabla} p$ to fit the LBM hypotheses and the resistivity definition.

\subsubsection{Tensorial simulated verification}

A sample is put in our numerical tube and the 630 Astivity calculated. Next, the sample is turned $90^{\circ}$, once again $90^{\circ}$, and once again by $90^{\circ}$. The directions are called respectively, $1,2,3$, and 4 . Table 2 shows the results obtained. They conform to the theory stating that if the pressure gradient is in an exactly inverted velocity field.

\subsubsection{Velocity fields}

As mentioned before, the visualization of the velocity field is also followed up from time to time. 
Table 2: Porosity in opposite directions, Dir3 $=-\operatorname{Dir} 1$ and Dir4 $=-$ Dir2.

\begin{tabular}{|c|c|c|c|}
\hline & Resistivity & & Resistivity \\
\hline Dir1 & $1.5225 \mathrm{e}+03$ & Dir2 & $1.6726 \mathrm{e}+03$ \\
\hline Dir3 & $1.5226 \mathrm{e}+03$ & Dir4 & $1.6724 \mathrm{e}+03$ \\
\hline
\end{tabular}

What is observed is that iteration by iteration the streamlines re-enforce up to convergence. Fig. 14 shows theses streamlines (in blue).



Figure 14: Flow field streamlines through a porous sample.

\subsection{Resistivity estimation results}

According to previous sections, a set of 10 slices is analyzed, extracted from the same set of slices used to estimate the porosity. The first slice, numbered 150 , is selected; next, the slices 200,300,..., 900 and 939 (the last one) are selected. For each slice, the resistivity is calculated in both directions 1 and 2 .

The results are reported in Table 3, where the lowest and highest value are, respectively, 853.4Pa.s $/ \mathrm{m}^{2}$ and 2242.8 Pa.s $/ \mathrm{m}^{2}$. The label Res refers to the resistivity of a slice as the mean value of the resistivity tensor: Res $=(\operatorname{Res} 1+\operatorname{Res} 2) / 2$. The highest difference between slices is obtained considering the slice $150\left(2165\right.$ Pa.s $\left./ \mathrm{m}^{2}\right)$ and the slice 800 $\left(1091\right.$ Pa.s $\left./ \mathrm{m}^{2}\right)$. This difference is of 1074 Pa.s $/ \mathrm{m}^{2}$. The slice giving the largest difference from one direction to another is the slice 700 , with a difference of $656 \mathrm{~Pa} . \mathrm{s} / \mathrm{m}^{2}$. As it will be explained in the following paragraph, these differences are not significant in terms of engineering acoustics; however, as we are in the case of very low resistivity, such differences may turn the value of resistivity from one slice to another of a single slice to double. The fundamental reason for that, to our view, is that a single slice is not representative of the actual representative volume. If the representative volume were the smallest possible representative volume, all the 790 slices available should be considered. That is why it is interesting to notice that the statistic mean value obtained of $1382 \pm 12\left(\right.$ Pa.s $\left./ \mathrm{m}^{2}\right)$, using this set of 10 slices, already gives a close approximation to the measured reference value of $1350 \mathrm{~Pa} . \mathrm{s} / \mathrm{m}^{2}$, obtained using a resistivity-meter. The reference values are labeled as RefTest in Table 3 .

Ten samples is a low number in terms of usual statistics. Therefore, aiming to confirm the results obtained, the analysis is extended by considering two larger set of slices: the first one with 17 slices, namely $150,200,250, \ldots$, and the second one with 33 slices, namely $150,175,200,225, \ldots, 939$. Extremal values of $3487 \mathrm{~Pa} . \mathrm{s} / \mathrm{m}^{2}$ (slice 275 ) and of 776 Pa.s $/ \mathrm{m}^{2}$ (slice 750 ) are encountered without significantly modifying the mean values reported in Table 4 The standard error also converges, which reinforces the results. The porosity is also analyzed, and no relation is found between the variation in porosity, from one slice to another, and the corre690 sponding variation in resistivity.

Table 3: LBM analysis of a sample of 10 slices, labeled according to the tomographic imaging order, and their respective porosities, resistivities Res 1 and Res 2 in directions 1 and 2, mean values of each resistivity tensor Res $=$ $(\operatorname{Res} 1+\operatorname{Res} 2) / 2$, the resulting Mean porosity and Res, and the measured values RefTest. Resistivities in $P a . s / m^{2}$.

\begin{tabular}{|c|c|c|c|c|}
\hline Slice & Porosity & Res 1 & Res 2 & Res \\
\hline 150 & 0.842 & 2242.8 & 2086.4 & 2165 \\
\hline 200 & 0.857 & 1264.1 & 1360.6 & 1312 \\
\hline 300 & 0.844 & 1690.2 & 1490.1 & 1590 \\
\hline 400 & 0.873 & 1229.0 & 942.9 & 1086 \\
\hline 500 & 0.868 & 1066.4 & 1335.2 & 1201 \\
\hline 600 & 0.862 & 1081.8 & 1318.7 & 1200 \\
\hline 700 & 0.861 & 2089.0 & 1432.5 & 1761 \\
\hline 800 & 0.865 & 1126.6 & 1056.0 & 1091 \\
\hline 900 & 0.864 & 853.4 & 1380.0 & 1117 \\
\hline 939 & 0.867 & 1193.0 & 1402.8 & 1298 \\
\hline Mean & $0.860 \pm 0.009$ & $1382 \pm 12$ \\
\hline RefTest & \multicolumn{6}{|c|}{0.866} & 1350 \\
\hline
\end{tabular}


Table 4: LBM analysis of sets of 10, 17 ans 33 slides with the respective mean porosities and mean $R e s$, and the measured values RefTest. Resistivities in $\mathrm{Pa} . \mathrm{s} / \mathrm{m}^{2}$.

\begin{tabular}{|c|c|c|}
\hline Number of Slices & Mean Porosity & Mean Res \\
\hline 10 & $0.860 \pm 0.009$ & $1382 \pm 12$ \\
\hline 17 & $0.863 \pm 0.003$ & $1336 \pm 09$ \\
\hline 33 & $0.863 \pm 0.003$ & $1370 \pm 07$ \\
\hline RefTest & 0.866 & 1350 \\
\hline
\end{tabular}

\subsection{Discussion}

To deepen into the analysis of the results, several considerations are made in the following, which are important to illustrate and contextualize this investigation.

\subsubsection{Sensitivity to global acoustic indicators}

The airflow resistivity is a parameter that covers a large range of values, generally from 10,000 to 100,000 Pa.s $/ \mathrm{m}^{2}$, for common acoustic materials. Thus, with an estimate mean resistivity of $1,382 P a . s / m^{2}$, calculated from actual values ranging from 853.4 to $2,242.8$ Pa.s $/ 2$ (see Table 3), our sample situates in the range of very low resistivities. It is shown in this part that, sighted from the two usual main global acoustic indicators - absorption and transmission loss (TL) - the seemingly large range of values should be considered actually close.

For example, a resistivity of 1,000 Pa.s $/ \mathrm{m}^{2}$ would produce quasi-identical absorption and TL curves compared to the ones produced by a resistivity of $2,000 P a . s / \mathrm{m}^{2}$, while a resistivity of $20,000 P a . s / m^{2}$ would produce very different results than a resistivity of $40,000 P a . s / \mathrm{m}^{2}$, although the proportion involved is the same for both cases.

The formulae of the JCA fluid equivalent model described in paragraph 3.2 are implemented in the semi-analytical Matlab transfer matrix code named TMTX. Fig. 15 and Fig. 16 show, respectively, the abortion and the TL curves obtained using TMTX when various resistivities are considered. The other parameters of the simulation are: $\phi=0.86, \alpha_{\infty}={ }_{735}$ $1, \Lambda=167.3 \mu \mathrm{m}$ and $\Lambda^{\prime}=334.6 \mu \mathrm{m}$. The thickness of the sample is $40 \mathrm{~mm}$, and normal incident acoustic waves are considered. For the range of variation of our parameter (Min, Nominal, Max), the curves are, indeed, superimposed.

\subsubsection{Representative volume}

The results presented in Table 3 indicate that, for a given slice, the resistivity calculated in direction



Figure 15: Absorption curves for $\sigma=10,000(\operatorname{sig} 10), \sigma=$ $20,000$ (sig20), $\sigma=5,000$ ( $\operatorname{sig} 5), \sigma=853.4$ (Min), $\sigma=$ $2,242.8$ (Max) and $\sigma=1,382$ (Nominal).



Figure 16: Transmission Loss curves for $\sigma=10,000$ ( $\operatorname{sig} 10$ ), $\sigma=20,000(\operatorname{sig} 20), \sigma=5,000$ (sig5), $\sigma=853.4$ (Min), $\sigma=2,242.8$ (Max) and $\sigma=1,382$ (Nominal).

1 is close to the one calculated in direction 2 , while the difference in resistivity from one slice to another is larger. From our point of view, this is due to two combined facts: i) the fact, already mentioned in paragraph 5.1, that the manufactured process tends to layer the material, and ii) the fact that the tomography images are also acquired in the plane of this slightly layered material. Finally, our material is slightly orthotropic. The slice producing the larger resistivity is the slice 150 , which is the 740 slice shown in Fig. 6. The slice producing the lowest resistivity is the slice 900, which is the image used in Fig. 14. It can be seen that the slice producing the larger resistivity is the slice with more fiber obstacles. 

implementation to characterize other fibrous mate- 830 rials.

\section{Acknowledgments}

The authors would like to thank the CAPESCOFECUB Project 773/13 - Proc. 8909-14-8, the CNPq/Universal Proc. 82351/2013-6 and the
CAPES/PNPD Proc. 1564115 for their financial support. We would also like to thank Jean-Daniel Chazot, from the Laboratoire Roberval (UMR CNRS 7337) of the Université de Technologie de Compiègne (UTC)/France, and Benjamin Smaniotto, Technical Manager of the tomograph of the Laboratoire de Méchanique et Technologie (LMT, UMR CNRS 8535) of the ENS Paris-Saclay, for their valuable help.

\section{Appendix A. LBM-BGK method}

The Lattice Boltzmann Method (LBM) can be seen as a spacial and temporal finite difference method which takes benefit of a mesh (the lattice) that has to be strictly constructed with square elements with $\Delta x=\Delta y=\Delta t$ for each element. Doing this, it is possible to solve the Boltzmann transport equation

$$
\frac{\partial f}{\partial t}+\vec{v} \cdot \vec{\nabla} f=\Omega
$$

This equation is an equilibrium equation where $f(\vec{x}, t)$ is the particle distribution function, $\vec{v}(\vec{x}, t)$ is the particle velocity, and $\Omega$ is the collision operator. The equilibrium is found iteratively. The main references used in this section are [40, 41, 42].

\section{Appendix A.1. Streaming and equilibrium}

Using the D2Q9 model, shown in Fig. A.17 the particle is restricted to stream in 9 possible directions described by the microscopic velocities $\vec{e}_{i}$, where the index $i \in\{0,1,2, \ldots 8\}$, with $i=0$ being attributed to the particle at rest. For each direction is also associated a probability function $f_{i}$. As important remarks: the vectors $\vec{e}_{i}$ do not change during the process, in opposition to their associated distribution functions; the vectors $\vec{e}_{i}$ on the diagonals are of $\sqrt{2}$ length, while the others are unitary.

The macroscopic fluid density $\rho(\vec{x}, t)$ and the macroscopic velocity $\vec{v}(\vec{x}, t)$ at each Lattice node can be extracted from the probability distribution functions of the particle by the equations

$$
\rho=\sum_{i} f_{i}, \quad \rho \vec{v}=\sum_{i} f_{i} \vec{e}_{i}
$$

and the pressure $p$ can be evaluated from the density as $p=\rho c_{s}^{2}$, where $c_{s}=1 / \sqrt{3}$ is the lattice speed of sound. 


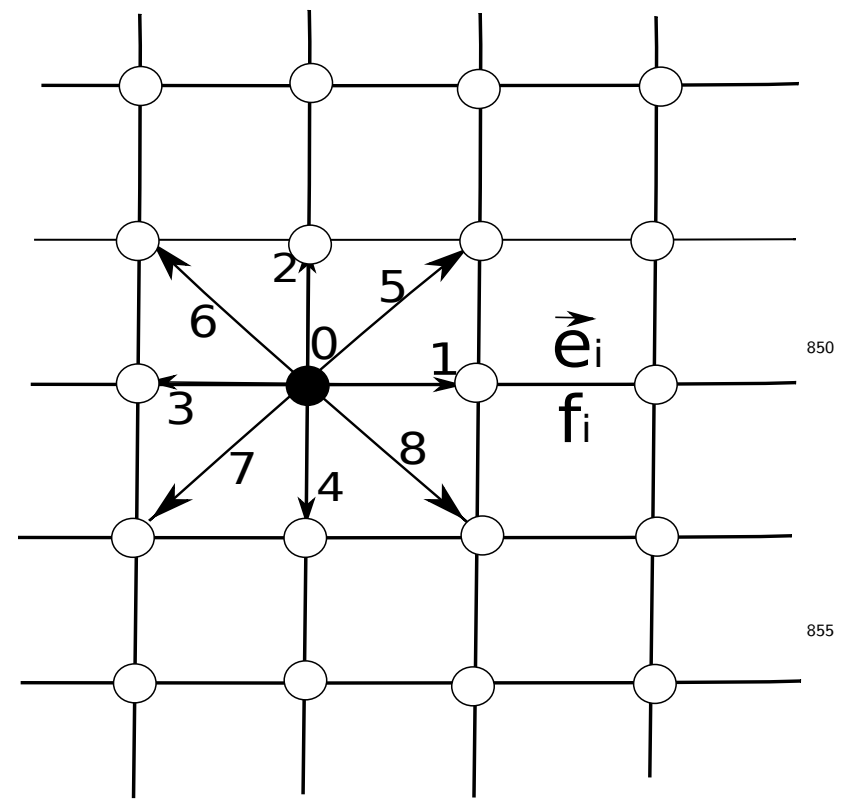

Figure A.17: D2Q9 discretization scheme.

Using the D2Q9 discretization and the

Bhatnagar-Gross-Krook (BGK) collision model (that suffices for laminar single phase flows), Eq. A.1 can be rewritten as

$f_{i}\left(\vec{x}+c \vec{e}_{i} \Delta t, t+\Delta t\right)-f_{i}(\vec{x}, t)=-\frac{f_{i}(\vec{x}, t)-f_{i}^{e q}(\vec{x}, t)}{\tau}$, 865

with $\tau$ being a relaxation time, and $f_{i}^{e q}$ the equilibrium distribution. Reorganizing Eq. A.3 allows for the calculation of the distribution at $t+\Delta t$ from ${ }^{870}$ the distribution at $t$ as

$f_{i}\left(\vec{x}+c \vec{e}_{i} \Delta t, t+\Delta t\right)=f_{i}(\vec{x}, t)-\frac{f_{i}(\vec{x}, t)-f_{i}^{e q}(\vec{x}, t)}{\tau}{ }_{875}$

$f_{i}^{e q}$ is evaluated from the macroscopic density and velocity as

$f_{i}^{e q}(\vec{x}, t)=\omega_{i} \rho\left(1+3 \frac{\vec{e}_{i} \cdot \vec{v}}{c^{2}}+\frac{9}{2} \frac{\left(\vec{e}_{i} \cdot \vec{v}\right)^{2}}{c^{4}}-\frac{3}{2} \frac{\vec{v} \cdot \vec{v}}{c^{2}}\right)$,

where $\omega_{i}$ are weight factors: $\omega_{i}=4 / 9$ for $i=0$, $\omega_{i}=1 / 9$ for $i=1,2,3,4$ and $\omega_{i}=1 / 36$ for $i=885$ $5,6,7,8$. In the previous equation $c=\Delta x / \Delta t$ is the lattice speed, which is taken to be 1 .
Another relation links the fluid kinematic viscosity $\mu$ to the relaxation time $\tau$ by

$$
\mu=\frac{2 \tau-1}{6} \frac{(\Delta x)^{2}}{\Delta t}
$$

The first term of the second member of Eq. A.4 refers to the streaming phase, while the second one to the collision phase. These two phases are generally separated in the programming process.

\section{Appendix A.2. Boundary conditions}

Imposing boundary conditions within the LBM is not a straightforward process because the macroscopic Navier-Stokes boundary conditions must be translated to microscopic boundary conditions on $f_{i}$ and $f_{i}^{e q}$. Boundary conditions are generally of the following types:

- Bounce Back: No-Slip Boundary Conditions;

- Boundary Conditions with Known Velocity;

- Periodic Boundary Conditions;

- Imposed Pressure Difference Boundary Conditions.

Our code is strictly limited to the resolution of our given problem. It is described, hereafter, the two types of boundary conditions considered: i) a Bounce Back scheme and ii) imposed body forces (which is a non standard type) of constant value and orientation.

i) Bounce back. It concerns the reflection of the particles on the fixed obstacles (the fibers of the porous samples). A full-way model of bounce-back is preferred to a mid-way one since the first does not require to know the normal orientation when a particle enters in contact with a rigid boundary. This model is adapted when several obstacles are present. At a first step, the fixed lattice nodes in contact with the wet area are to be considered in the streaming process. Next, the particle distribution functions of theses nodes, which point to the exterior of the fluid area, are reversed in direction.

ii) Body forces. We intend, from the definition of the permeability (Eq. 7), to impose a pressure gradient (a body force) all over the fluid domain. This leads to the advanced developments studied in 42 because the body forces have to be added to the initial equilibrium Eq. A.3. 


$$
\begin{aligned}
f_{i}\left(\vec{x}+c \vec{e}_{i} \Delta t, t\right. & +\Delta t)-f_{i}(\vec{x}, t)= \\
& -\frac{f_{i}(\vec{x}, t)-f_{i}^{e q}(\vec{x}, t)}{\tau}+\Delta t F_{i},
\end{aligned}
$$

where $F_{i}$ are the body forces.

Theoretically, all previous formulae A.1 to A.5

should be modified. However, 42 shows that when the body forces are slightly changing in space and time, the representation of the body as $F_{i}=$ $\omega_{i} \vec{e}_{i} \cdot \vec{F} / c_{s}^{2}$ can be directly introduced in the previous model unaltered, and the Navier-Stokes equations remain verified.

\section{Appendix A.3. Algorithm}

Finally, using the notation := as the programming affectation operator, our algorithm for a cur- 950 rent iteration is the following:

- $f_{i}($ iter +1$):=\operatorname{Pr}\left(f_{i}(\right.$ iter $\left.)\right)$ (propagation);

- $f_{i}($ iter +1$):=f_{i}($ iter +1$)+F_{i}$, (body forces $)$;

- $f_{i}($ iter +1$):=B b\left(f_{i}(\right.$ iter +1$\left.)\right)$ (bounce-back);

- $\rho:=\sum_{i} f_{i}($ iter +1$) ; \quad \vec{v}:=\sum_{i} f_{i}($ iter +1$) \vec{e}_{i} / \rho 960$ (macro);

- $f_{i}^{e q}(\rho, \vec{v})$ (equilibrium);

- $f_{i}($ iter +1$):=f_{i}($ iter +1$)-\frac{f_{i}(i t e r+1)-f_{i}^{e q}}{\tau}($ collision).

\section{References}

\section{References}

[1] M. A. Biot, Theory of propagation of elastic waves in a fluid-saturated porous solid. I. Low-frequency range. II. Higher frequency range, JASA 28 (1956) 168-191. doi:10.1121/1.1908239,10.1121/1.1908241

[2] J. F. Allard, N. Atalla, Propagation of Sound in Porous Media: Modeling Sound Absorbing Materials, 2nd Edition, Wiley, 2009.

[3] K. V. Horoshenkov, A review of acoustical methods for porous material characterization, International Journal of Acoustics and Vibration 22 (2017) 92-103. doi:10. 20855/ijav.2017.22.1455

[4] Z. L. Liu, Multiphysics in Porous Materials, Springer, 2018. doi:10.1007/978-3-319-93028-2

[5] O. Doutres, Y. Salissou, N. Atalla, R. Panneton, Evaluation of the acoustic and non-acoustic properties of sound absorbing materials using a three-microphone ${ }^{9}$ impedance tube, Applied Acoustics 71 (2010) 506-509. doi:10.1016/j.apacoust.2010.01.007
[6] F. C. Bannwart, L. F. Cóser, B. N. Huallpa, D. A. Siviero, J. F. R. Arruda, On the effects of air cavity backing porous materials for its characterization by the three-microphone method, in: Proc. of the 34th Int. Conf. on Noise and Vibration Engineering (ISMA2016), Leuven, Belgium, September 19-21, 2016, pp. 33-46. doi:10.13140/RG.2.2.24667.36642

[7] A. Abbad, N. Atalla, M. Ouisse, O. Doutres, Numerical and experimental investigations on the acoustic performances of membraned helmholtz resonators embedded in a porous matrix, Journal of Sound and Vibration 459 (2019) 1-17. doi:10.1016/j.jsv.2019.114873

[8] Y. Atalla, R. Panneton, Low frequency inverse method for the identification of the viscous and thermal characteristic lengths of porous media, in: Int. Congress on Noise Control Engineering, Fort Lauderdale, Florida, U.S.A., December 06-08, 1999.

[9] O. Doutres, Y. Salissou, N. Atalla, R. Panneton, Inverse acoustical characterization of open cell porous media using impedance tube measurements, in: Can. Acoust., Vol. 33, 2005, pp. 11-24.

[10] M. Guédra, F. C. Bannwart, G. Penelet, P. Lotton, Parameter estimation for the characterization of thermoacoustic stacks and regenerators, Applied Thermal Engineering 80 (2015) 229-237. doi:10.1016/j. applthermaleng.2015.01.058.

[11] T. Zielinski, Normalized inverse characterization of sound absorbing rigid porous media, JASA 137 (2015) 3232-3243. doi:10.1121/1.4919806.

[12] Y. Yang, Z. Chen, A model for calculating the air flow resistivity of glass fiber felt, Applied Acoustics 91 (2015) 6-11. doi:10.1016/j.apacoust.2014.11.006

[13] A. I. Hurrell, K. V. Horoshenkov, M. T. Pelegrinis, The accuracy of some models for the airflow resistivity of nonwoven materials, Applied Acoustics 130 (2018) 230237. doi:10.1016/j.apacoust.2017.09.024.

[14] P. Soltani, E. Taban, M. Faridan, S. E. Samaei, S. Amininasab, Experimental and computational investigation of sound absorption performance of sustainable porous material: Yucca gloriosa fiber, Applied Acoustics 157 (2019) 1-12. doi:10.1016/j.apacoust.2019. 106999

970 [15] G. Ji, J. Cui, Y. Fang, S. Yan, J. Zhou, J.-K. Kim, Nano-fibrous composite sound absorbers inspired by owl feather surfaces, Applied Acoustics 156 (2019) 151157. doi:10.1016/j.apacoust.2019.06.021

[16] L. C. G. Pennafort, Aplicação da mecânica da danificação na análise do comportamento de materiais compósitos poliméricos reciclados reforçados por fibras de coco, Ph.D. thesis, Universidade Federal de Ceará (2014).

[17] H. Taud, R. Martinez-Angeles, J. F. Parrot, L. Hernandez-Escobedo, Porosity estimation method by x-ray computed tomography, J. Petroleum Sci. and Eng. 47 (2005) 209-217. doi:10.1016/j.petrol.2005. 03.009

[18] P. G. K. Gao, J. A. W. van Dommelen, M. G. D. Geers, Computational homogenization of sound propagation in a deformable porous material including microscopic viscous-thermal effects, Journal of Sound and Vibration 365 (2016) 119-133. doi:10.1016/j.jsv.2015.11.037

[19] T. Zielinski, Microstructure representations for sound absorbing fibrous media: $3 \mathrm{~d}$ and $2 \mathrm{~d}$ multiscale modelling and experiments, Journal of Sound and Vibration 409 (2016) 112-130. doi:10.1016/j.jsv.2017.07.047 
[20] J. Y. Chung, D. A. Blaser, Transfer function method of measuring in-duct acoustic properties. ii. experiment, JASA 68 (1980) 914-921. doi:10.1121/1.384779.

[21] O. Tanneau, J.-B. Casimir, P. Lamary, Optimization of multilayered panels with poroelastic components for an acoustical transmission objective, JASA 120 (2006) 1227-. doi:10.1121/1.2228663

[22] B. Brouard, B. Castagnède, M. Henry, D. Lafarge, 1065 S. Sahraoui", Mesure des propriétés acoustiques des matériaux poreux, Techniques de l'ingénieur Acoustique : mesures, contrôle, applications base documentaire : TIB423DUO. (ref. article : r6120), fre. arXiv: basedocumentaire:TIB423DUD.

[23] N. A. O. Doutres, H. Osman, Transfer matrix modeling and experimental validation of cellular porous material with resonant inclusions, JASA 137 (2015) 3502-3513. doi:10.1121/1.4921027

[24] O. Tanneau, P. Lamary, Y. Chevalier, A boundary ele- 1075 ment method for porous media, JASA 120 (2006) 1239-. doi:10.1121/1.2228663

[25] P. Lamary, R. de Aráujo Bezerra, I. da Silva Júnior, E. D. de Pontes, A. Benallal, A multi-physic implementation of the finite element method applied to 1080 research on acoustic poro-elastic materials, in: CILAMCE 2014, Fortaleza, 2014, 10.13140/2.1.2515.2327. doi:10.13140/2.1.3301.6644.

[26] R. Rumpler, Efficient finite element approach for structural-acoustic applications including 3d modelling of sound absorbing porous materials, Ph.D. thesis, KTH, MWL Numerical acoustics, qC 20120224 (2012).

[27] N. Dauchez, P.-A. Yvars, Inverse method for porous material characterization using the constraint satisfaction problem approach, in: Acoustics 2012, Nantes, 2012.

[28] AFPC-AFREM, Détermination de la masse volumique apparente et de la porosité accessible à l'eau - méthodes recommandées pour la mesure des grandeurs associées à la durabilité, in: Compte-rendu des Journées Techniques, Toulouse, 11-12 Décembre 1997, 1997, pp. 121124 .

[29] Y. Salissou, R. Panneton, Pressure/mass method to measure open porosity of porous solids, J. Appl. Phys. 101, 2007, 124913: 1-7. doi:10.1063/1.2749486

[30] A. Keller, High resolution, non-destructive measurement and characterization of fracture apertures, Int. Journal Rock Mech. Min. Sci. 35(8) (1998) 1037-1050. doi:10.1016/S0148-9062(98)00164-8

[31] T. W. Ridler, S. Calvard, Picture thresholding using an iterative selection method, IEEE Transactions on Systems, Man and Cybernetics 8 (1978) 630-632. doi: 10.1109/TSMC.1978.4310039

[32] L. K. Huang, M. J. J. Wang, Image thresholding by minimizing the measures of fuzzinesss, Pattern Recognition vol 23 No. 1 (1995) 41 - 51. doi:10.1016/ 0031-3203(94) E0043-K

[33] C. H. Li, C. K. Lee, Minimum cross entropy thresholding, Pattern Recognition 26, No 4 (1993) 617-625. doi:10.1016/0031-3203(93) 90115-D

[34] C. A. Glasbey, An analysis of histogram-based thresholding algorithms, CVGIP: Graphical Models and Image Processing 55 (1993) 532-537. doi:10.1006/cgip. 1993.1040

[35] W. H. Tsai, Moment-preserving thresholding: a new approach, Computer Vision, Graphics, and Image Processing 29 (1985) 377-393. doi:10.1016/
0734-189X (85) 90133-1

[36] N. Otsu, A tlreshold selection method from gray-level histograms, IEEE Transactions on systems, Man, and

Cybernetics SMC-9, No. 1, January (1979) 62-66. doi: 10.1109/TSMC.1979.4310076

[37] A. G. Shanbhag, Utilization of information measure as a means of image thresholding, CVGIP: Graphical Models and Image Processing vol 56 No 5 (1994, September) 414-419. doi:10.1006/cgip.1994.1037

[38] C. V. der Kelena, P. Göransson, Identification of the full anisotropic flow resistivity tensor for multiple glass wool and melamine foam samples, JASA 134 (2013) 4659. doi:10.1121/1.4824841

[39] T. Krüger, Unit conversion in lbm, in: LBM Workshop, Edmonton, Canada, 2011.

[40] Y. Bao, J. Meskas, Lattice boltzmann method for fluid simulations, Tech. rep., New York University,Department of Mathematics (2011).

[41] P. Wang, Lattice boltzmann simulation of permeability and tortuosity for flow through dense porous media, Mathematical Problems in Engineering Article ID 694350 (2014). doi:10.1155/2014/694350.

[42] Z. Guo, C. Zheng, B. Shi, Discrete lattice effects on the forcing term in the lattice boltzmann method, PHYSICAL REVIEW E 65, 046308 (2002). doi:10.1103/ PhysRevE.65.046308 